malignancy, are managed. Some patients are referred to liver clinic due to elevated fibrosis scores as a result of other pathologies, and the enhanced liver fibrosis (ELF) test has recently been added to our algorithm to further stratify those who require liver services.

\section{PWE-8 ALBUMIN BILIRUBIN SCORE FOR PREDICTING MORTALITY IN SUDANESE PATIENTS WITH ACUTE ON CHRONIC LIVER FAILURE}

${ }^{1}$ Esraa Barri*, ${ }^{2}$ Omer Kheir, ${ }^{1}$ Salma Barakat. ${ }^{1}$ Hepatology unit, NCGLD, Khartoum, Sudan; ${ }^{2}$ Research unit, NCGLD, Khartoum, Sudan

10.1136/gutjpl-2021-BSG.198

Introduction The albumin-bilirubin (ALBI) score, a new model for assessing severity of liver dysfunction was initially used in hepatocellular carcinoma but has not been thoroughly investigated in ACLF. The aim of this study was to assess prognostic value of ALBI score for predicting 28 days mortality in Sudanese patients with ACLF.

Methods This cross-sectional study included 34 patients diagnosed as ACLF using APASL criteria for diagnosis. The etiology of the underlying chronic liver disease and precipitating cause of ACLF were investigated and ALBI, MELD and CTP scores were calculated within 24 hours of admission. Mortality was assessed during hospital stay and if discharged at 28 days post enrollment by telephone conversation. Statistical analysis was performed using Statistical Package for the Social Sciences (SPSS 2.0).

Results ALBI scores were significantly associated with 28-days outcome as the mean of ALBI in the non-survived patients was higher than in the survived $(-0.45 \pm 0.39$ vs $-0.65 \pm 0.59$; P. value $=0.004)$. Regarding the predictive performances of the ALBI, MELD and CTP scores in detecting the 28-days mortality, ROC analysis showed that the AUC of MELD was 0.746 (95\% CI: $0.575-0.916 ;$ P. value $=0.015)$, ALBI was

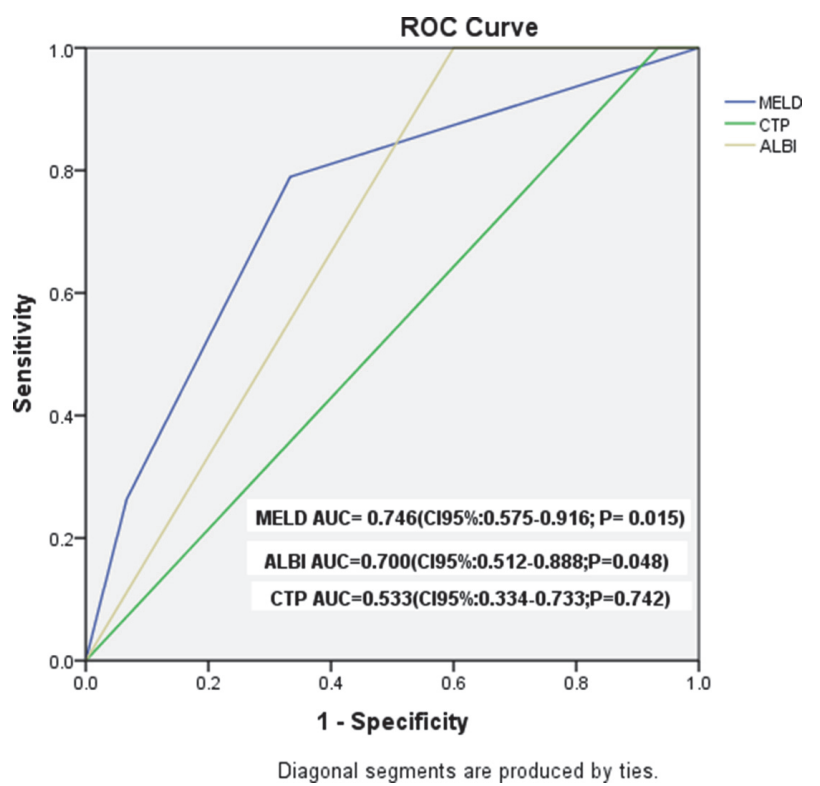

Abstract PWE-8 Figure 1 The multiple receiver operating characteristic curve (ROC) to evaluate validity of MELD, ALBI and CTP in detecting the 28-days mortality
0.700 (95\% CI: $0.512-0.888 ;$ P. value $=0.048)$, and CTP was 0.533 (95\% CI: 0.334-0.733; P. value $=0.742$ )

Conclusion A higher ALBI score measured at admission is a useful predictor of 28 day mortality in ACLF patients. ALBI score was comparable to MELD and did better than CTP scores in predicting short-term mortality in these patients.

\section{PWE-9 MISSED OPPORTUNITIES FOR EFFECTIVE ALCOHOL SCREENING IN THE EMERGENCY DEPARTMENT - TIME TO ACT?}

Neil Gordon*, Jill Harrison, Rebecca Fennell, Dianne Backhouse, George Abouda, Lynsey Corless. Hull University Teaching Hospitals Nhs Trust, Hull, UK

\subsection{6/gutjnl-2021-BSG.199}

Introduction Alcohol Care Teams (ACT) are integral to bestpractice management of people with Alcohol Use Disorders (AUD), the consequences of which represent a significant proportion of in-patient hepatology activity in the UK. The ACT in our centre was established in January 2020. It comprises two clinical nurse specialists (CNS) providing comprehensive alcohol assessments and psychological interventions, supervised medical withdrawal and fibrosis screening, and a communityled, hospital-based addiction counselling service (ReNew), and is supported by 2 consultants. The CNS service is funded to provide only weekday cover $(8 \mathrm{am}-5 \mathrm{pm})$ and there is currently no formal alcohol screening process in the Emergency Department (ED), potentially leading to missed opportunities for intervention. We therefore performed an audit to determine effectiveness of AUD screening in ED.

Methods A retrospective analysis of all ED attendances from $6 / 6 / 20$ to 6/9/20 was undertaken. Visits coded with a diagnosis wholly attributable to alcohol were identified as opportunities for AUD screening. Records were reviewed for presence/ absence of AUD screening (specifically scores such as AUDIT), and if a referral was made to ACT. Data was also collected on how many attendances resulted in hospital admission, and if ACT CNS review took place at any time during that period. A secondary analysis was performed looking specifically at frequent attenders (patients who had attended ED $>10$ times in 12 months).

Results 462 unique attendances (326 patients) were recorded, with 'Intoxicated' the most frequent reason coded. Only 8 attendances (1.7\%) were referred to ACT, and just $1(0.2 \%)$ had documented evidence of formal alcohol screening. 365 (79\%) of attendances were at weekends/out-of-hours, most of which is not covered by current ACT funding, and this may partly explain the very low referral rates. There were 175 (38\%) admissions to a medical or short stay area. Of those, $55(31 \%)$ were seen by ACT. Repeat attendances were common: 26 patients had attended ED $>10$ times in the last year (mean=19/patient) and worryingly, $46 \%$ of those had never been reviewed by ACT CNS. 35\% of patients who did receive ACT input for medically-assisted withdrawal remained abstinent at 1 month, suggesting considerable potential to improve abstinence rates across our population by increasing ACT referrals.

Conclusions This audit illustrates the consequences of inadequate AUD screening in ED, and subsequent lost opportunities to signpost the majority who do not require admission, to community alcohol services; namely high rates of hospital admissions, frequent attendances, and greater numbers of 